\title{
Synaptic patterns of rye B chromosomes. IV. The B isochromosomes
}

\author{
J. L. SANTOS*, M. M. JIMÉNEZ \& M. DÍEZ \\ Departamento de Genética, Facultad de Biologia, Universidad Complutense de Madrid, 28040 Madrid, Spain
}

\begin{abstract}
Pairing of B isochromosomes in rye was studied by surface spreading of synaptonemal complexes in pollen mother cells from plants containing one small iso- $\mathrm{B}$ (iso- $\mathrm{B}^{\mathrm{S}}$ ), one large iso- $\mathrm{B}$ (iso- $\mathrm{B}^{\mathrm{L}}$ ), two iso- $\mathrm{B}^{\mathrm{L}}$, one iso- $\mathrm{B}^{\mathrm{S}}$ plus one standard $\mathrm{B}$ (iso- $\mathrm{B}^{\mathrm{S}}+\mathrm{st} \mathrm{B}$ ), one iso- $\mathrm{B}^{\mathrm{S}}$ plus two standard $\mathrm{Bs}$ (iso- $\mathrm{B}^{\mathrm{S}}+2$ $\mathrm{stB}$ ) and one iso- $\mathrm{B}^{\mathrm{L}}$ plus two standard $\mathrm{Bs}$ (iso- $\mathrm{B}^{\mathrm{L}}+2 \mathrm{stB}$ ). The main characteristics of iso-Bs were as follows. (1) Their location in the surface-spread nuclei was peripheral. (2) Their period of pairing relative to that of the A-set was delayed. (3) The commonest pachytene configuration was a symmetrical hairpin loop, although they could also undergo nonhomologous synapsis. (4) Synapsis could start at the chromosome ends, at the putative centromeric region or at both regions more or less simultaneously. (5) The frequency of iso- $\mathrm{B}^{\mathrm{L}}$ bivalents was only 46 per cent rather than the 66 per cent predicted by the random-end-pairing model. Interchromosomal pairing was frequent in these configurations. (6) In pairing competition situations involving three and four doses of the short arm of the standard $\mathrm{B}$ (iso- $\mathrm{B}^{\mathrm{S}}+\mathrm{stB}$, iso- $\mathrm{B}^{\mathrm{S}}+2 \mathrm{stB}$ ), a large predominance of self-synapsed iso- $\mathrm{B}^{\mathrm{S}}$ univalents ( 63 per cent and 80 per cent respectively) was found. On the other hand, there was no apparent pairing preference among four doses of the long arm of the standard $B$ in the iso$\mathrm{B}^{\mathrm{L}}+2 \mathrm{stB}$ combination. Similarities and differences between the synaptic behaviours of iso-Bs of Crepis capillaris and Secale cereale are discussed.
\end{abstract}

Keywords: B chromosomes, meiosis, pairing competition, rye, synapsis.

\section{Introduction}

Rye is one of the species that possesses a highly polymorphic B system. Besides the predominant standard B type (stB) there are several variants arising by simple rearrangements (Jones \& Rees, 1982). We are using this polymorphism as a tool to analyse different aspects of chromosome pairing in plants. In previous papers (Santos et al., 1993a; Díez et al., 1993; Jiménez et al., 1994) we studied the synaptic behaviour of the acrocentric stBs, and the deficient $B(\mathrm{~dB})$ that has lost the terminal half of the long arm of the stB. The present paper reports on the analysis of the pairing patterns displayed by the $\mathrm{B}$ isochromosomes (iso-Bs). These chromosomes originated by misdivisions at the centromere of the stBs giving rise to telocentrics which then form isochromosomes of the short (iso- $\mathrm{B}^{\mathrm{S}}$ ) and the long (iso- $\mathrm{B}^{\mathrm{L}}$ ) arms.

Müntzing \& Lima de Faria $(1949,1953)$ showed, by light microscopy, that the large and the small metacentric B chromosomes (Bs) of rye formed fold-back hairpin loops at pachytene, thus confirming the

\footnotetext{
*Correspondence.
}

isochromosome nature of these chromosomes. Surprisingly, the application of a surface spreading technique for making whole mount preparations of synaptonemal complexes (SCs) revealed that both stB and $\mathrm{dB}$ chromosomes could also display similar pairing configurations to those iso-Bs (Santos et al., 1993a; Jiménez et al., 1994). Our purpose in this paper is to reanalyse the primary synaptic behaviour of both types of iso-Bs, and to provide new insights into the pairing process of these chromosomes. Some situations of competitive pairing between iso-Bs and stBs are also studied.

\section{Materials and methods}

Six rye plants from progenies of different inbred lines crossed with the JNK cultivar carrying one iso- $\mathrm{B}^{\mathrm{S}}$ (two plants), one iso- $\mathrm{B}^{\mathrm{L}}$ (two plants) and two iso- $\mathrm{B}^{\mathrm{L}}$ (two plants), and three plants from the Korean Puyo population carrying 1 iso- $\mathrm{B}^{\mathrm{S}}+1$ stB (one plant), 1 iso- $\mathrm{B}^{\mathrm{S}}+2$ stB (one plant) and 1 iso- $\mathrm{B}^{\mathrm{L}}+2$ st $\mathrm{B}$ (one plant) were used.

Single anthers of the emerging spikes were squashed in 2 per cent acetic orcein to locate the stages of meiosis. Two sister anthers of the same floret at zygo- 
tene or pachytene were then prepared for SC isolation, as described by Holm (1986) with minor modifications (Santos et al., 1994). Surface-spread preparations were silver-stained by the method of Loidl (1984).

For metaphase I observations, the anthers were fixed in 1:3 acetic acid:ethanol and stored at $4^{\circ} \mathrm{C}$. The fixed material was squashed and C-banded using the Giemsa staining procedure of Giráldez et al. (1979).

A summary of the B chromosome constitution in the cells analysed is given in Table 1.

\section{Results}

\section{The small $B$ isochromosome (iso- $B^{S}$ )}

The iso- $\mathrm{B}^{\mathrm{S}}$ was analysed in 44 pachytene and three zygotene surface-spread nuclei from two plants (Table 1). The mean iso- $\mathrm{B}^{\mathrm{S}}$ axis length, when fully paired, was $13.92 \pm 0.73 \mu \mathrm{m}$, and the average percentage length of the $\mathrm{B}$ axis relative to that of the A-set SC length was about 2.6 per cent.

At pachytene, when the A complement is fully paired, the iso- $\mathrm{B}^{\mathrm{S}}$ is located peripherally in 95 per cent of the nuclei (42/44), and it displays eight different configurations (Fig. 1). The commonest form is a symmetrical univalent hairpin loop (A), that was observed in 29/44 nuclei (Fig. 2a). Centromere structures were not preserved in these surface-spread SC preparations but it is expected that centromeres are located at the points of inflexion of the hairpin loops in the iso- $\mathrm{B}^{\mathrm{S}}$ univalents and also in the iso- $\mathrm{B}^{\mathrm{L}}$ univalents described later. In configuration $\mathrm{D}$ the telomeric ends are independently synapsed with different interstitial regions. In the remainder of the configurations $\left(\mathrm{B}, \mathrm{B}^{\prime}\right.$, $\mathrm{C}, \mathrm{C}^{\prime}, \mathrm{E}$ and $\mathrm{F}$ ) the iso- $\mathrm{B}^{\mathrm{S}}$ is incompletely paired, and it follows that the period in which it achieves self-synapsis is delayed relative to that of the normal complement. The existence of one pairing initiation site is the most frequent situation, with the exceptions of configurations $\mathrm{D}$ and $\mathrm{E}$. There is no indication that pairing initiation occurs preferentially at or near telomeres (B and $\left.\mathrm{B}^{\prime}\right)$, or pericentromerically $\left(\mathrm{C}\right.$ and $\left.\mathrm{C}^{\prime}\right)$.

In one zygotene nucleus, the initiation of pairing was indicated by the presence of a short hairpin loop (Fig. $2 b$ ) that was not centrally placed in each chromosome $\mathrm{arm}$. Inequalities between axes were also found in some pachytene configurations $\left(\mathrm{B}^{\prime}, \mathrm{C}^{\prime}\right.$ and $\left.\mathrm{E}\right)$.

The frequencies of appearance of the iso- $\mathrm{B}^{\mathrm{S}}$ at metaphase I, as ring or rod univalents, could not be ascertained due to the small size of this chromosome and its high degree of condensation at this stage.

\section{The large $B$ isochromosome (iso- $B^{L}$ )}

Pairing of the iso- $\mathrm{B}^{\mathrm{L}}$ was analysed in 41 pachytene and 5 zygotene nuclei from two plants (Table 1). The mean axis length, when fully paired, was $69.6 \pm 1.15 \mu \mathrm{m}$. This measurement is in concordance with that of the iso- $\mathrm{B}^{\mathrm{S}}$ mentioned above and that of the standard $\mathrm{B}$, $43.5 \pm 0.8 \mu \mathrm{m}$ reported by Santos et al. (1993a). The average percentage length of the $\mathrm{B}$ axis relative to that of the A-set SC length was about 13 per cent.

At pachytene, the iso- $\mathrm{B}^{\mathrm{L}}$ is located peripherally in the spreads ( 34 of 41 nuclei), and shows delayed pair-

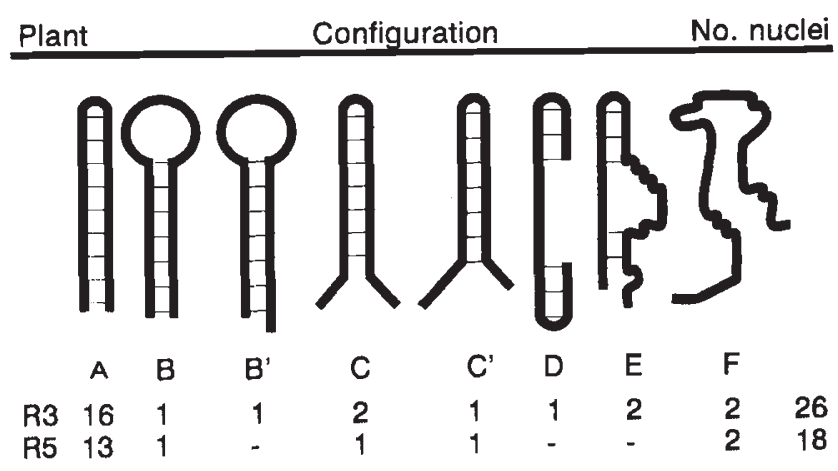

Fig. 1 Frequencies of the different pachytene configurations displayed by a single small B isochromosome.

Table 1 A summary of the B chromosome constitution in the rye cells analysed

\begin{tabular}{|c|c|c|c|c|}
\hline Plant & No. Bs & Pachytene & Zygotene & Metaphase I \\
\hline R3 & 1 iso- $B^{S}$ & 26 & 1 & - \\
\hline $\mathrm{R} 5$ & 1 iso- $B^{S}$ & 18 & 2 & - \\
\hline R11 & 1 iso- $\mathrm{B}^{\mathrm{L}}$ & 22 & 1 & 100 \\
\hline $\mathrm{R} 12$ & 1 iso- $\mathrm{B}^{\mathrm{L}}$ & 19 & 4 & 100 \\
\hline R21 & 2 iso- $B^{L}$ & 27 & - & 100 \\
\hline R26 & 2 iso- $\mathrm{B}^{\mathrm{L}}$ & 10 & - & 100 \\
\hline R35 & $1 \mathrm{stB}+1$ iso- $\mathrm{B}^{\mathrm{S}}$ & 27 & - & 100 \\
\hline R42 & $2 \mathrm{stB}+1$ iso $-\mathrm{B}^{\mathrm{s}}$ & 10 & - & 100 \\
\hline R51 & $2 \mathrm{stB}+1$ iso- $\mathrm{B}^{\mathrm{L}}$ & 22 & - & 100 \\
\hline
\end{tabular}


ing relative to the A-set in 20 per cent of the nuclei ( $8 /$ 41); see configurations B, C, D and G in Fig. 3. Among the other pachytene configurations (Fig. 3), the most frequent one is the expected symmetrical univalent hairpin loop (A), that was observed in $20 / 41$ nuclei
(Fig. 2c). Configurations $\mathrm{E}$ (Fig. 2d), $\mathrm{E}^{\prime}$ and $\mathrm{F}$ reveal different extents of nonhomologous synapsis and $\mathrm{B}, \mathrm{C}$ and $\mathrm{D}$ indicate different synaptic initiation patterns, namely: at the chromosome ends (C), at the point of inflexion of the hairpin loop, the putative centromeric
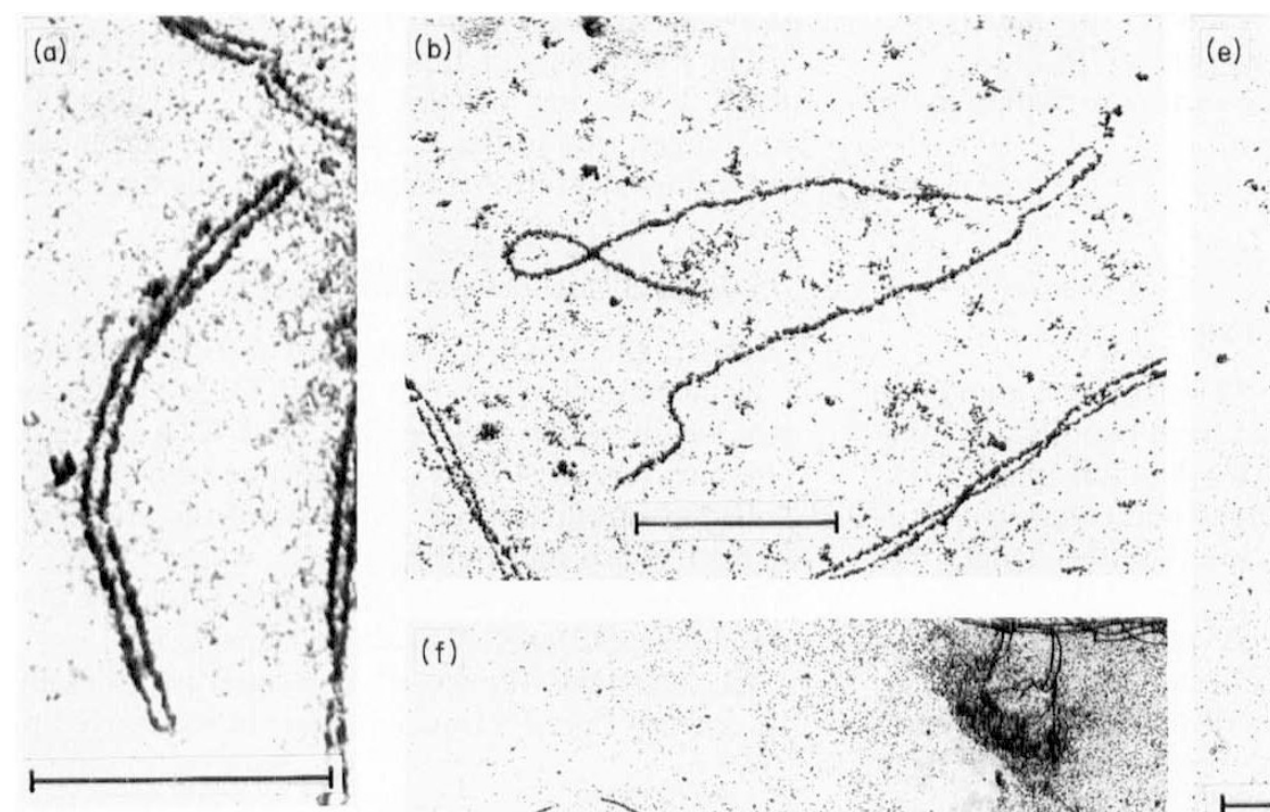

(f)
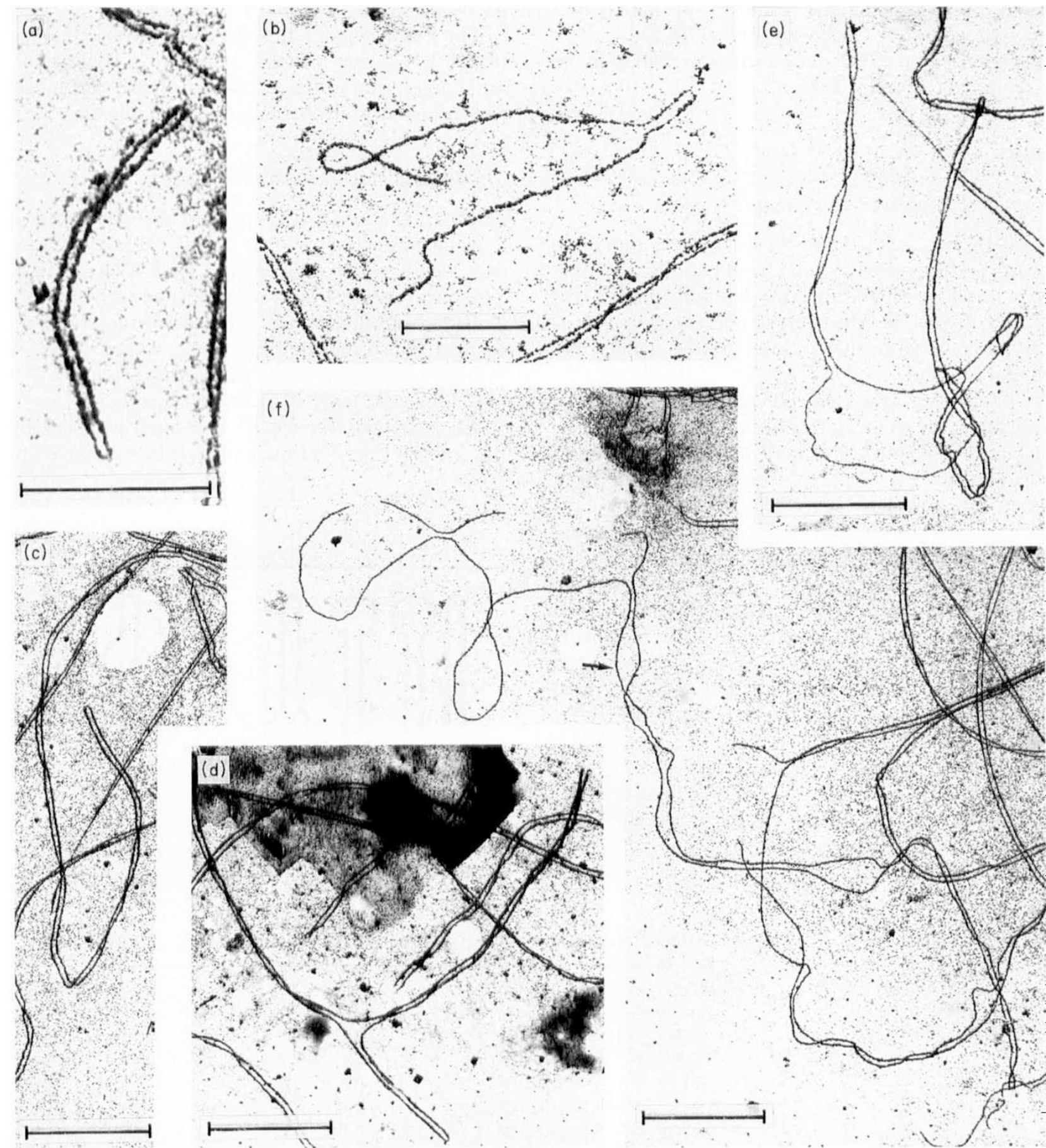

Fig. 2(a) Iso- $\mathrm{B}^{\mathrm{S}}$; pachytene symmetrical univalent hairpin loop. (b) Iso- $\mathrm{B}^{\mathrm{S}}$; zygotene univalent showing a short hairpin loop almost centrally located. (c) Iso-B ${ }^{\mathrm{L}}$; pachytene symmetrical univalent hairpin loop. (d) Iso- $\mathrm{B}^{\mathrm{L}}$; pachytene univalent showing nonhomologous synapsis. (e) Iso- $\mathrm{B}^{\mathrm{L}}$; pachytene univalent showing synapsis initiation at the chromosome end and at the putative centromeric region. (f) Iso- $\mathrm{B}^{\mathrm{L}}$; zygotene univalent (arrowed) showing four separate $\mathrm{SC}$ stretches.

Each scale bar represents $5 \mu \mathrm{m}$. 

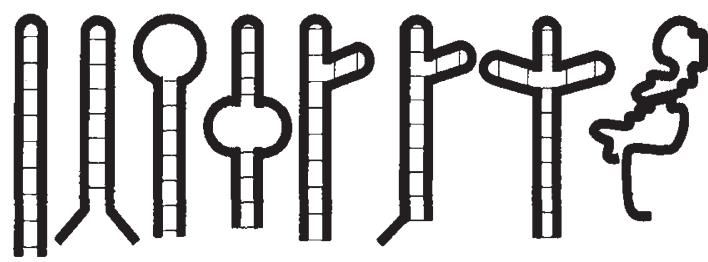

$\begin{array}{lccccccccc} & \text { A } & \text { B } & \text { C } & \text { D } & \text { E } & \text { E' } & \text { F } & \text { G } & \\ \text { R11 } & 9 & 1 & 1 & 2 & 5 & 1 & 1 & 2 & 22 \\ \text { R12 } & 11 & - & 1 & - & 3 & 1 & 2 & 1 & 19\end{array}$

Fig. 3 Frequencies of the different pachytene configurations displayed by a single large $B$ isochromosome.

region $(\mathrm{B})$, or at both regions more or less simultaneously (D, Fig. 2e).

Iso- $\mathrm{B}^{\mathrm{L}} \mathrm{s}$ less than 50 per cent paired invariably have two, three or four separate SC stretches without any consistent pattern of synapsis initiation (Fig. 2f). An analogous situation to that described for the iso- $\mathrm{B}^{\mathrm{S}}$ was also found, because irregularities between axes were observed in one zygotene nucleus and in some pachytene configurations $\left(\mathbf{E}^{\prime}\right)$.

The iso- $\mathrm{B}^{\mathrm{L}}$ appeared as an iso-ring univalent at metaphase I in 26 per cent $(26 / 100$, plant R11) and 13 per cent (13/100, plant R12) of the cells, values similar to the 17 per cent reported by Müntzing (1944) for an iso- $\mathrm{B}^{\mathrm{L}}$ of the rye variety 'Östgöta Gråråg'.

\section{Two large $B$ isochromosomes (2 iso- $\left.B^{L}\right)$}

Two iso- $\mathrm{B}^{\mathrm{L}}$ chromosomes were analysed in 37 pachytene nuclei from two plants (Table 1, Fig. 4).

Configuration A, a pair of hairpin looped univalents, results from pairing between the two arms of the same chromosome. It was observed in 54 per cent of nuclei $(20 / 37)$. A straight bivalent (B) is formed when exclusive interchromosomal pairing occurs between both iso- $\mathrm{B}^{\mathrm{L}} \mathrm{s}$. Configuration $\mathrm{C}$ is a slight modification of $\mathrm{B}$, the straight disposition of the axes being accompanied by intrachromosomal synapsis at some regions (Fig. 5a). Configuration $\mathrm{D}$ implies interchromosomal synapsis with a single pairing partner switch (PS). In three from 10 nuclei the PS is asymmetrically located with respect to chromosome ends (Fig. 5b) which indicates either the existence of repeat sequences or some stretches of nonhomologous synapsis.

The mean B bivalent frequency at metaphase I was 14 per cent (14/100 in plant R21) and 7 per cent (7/100 in plant R26), respectively.
Plant Configuration No. nuclei

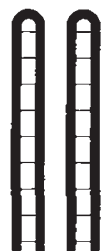

A

R21 14

R26 6

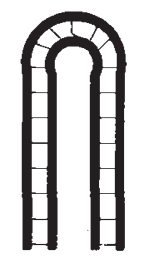

B

2

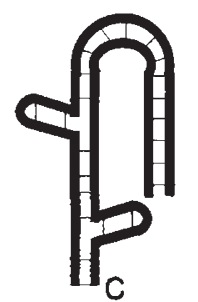

2

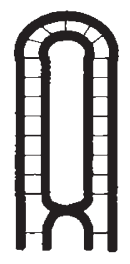

D

$\begin{array}{ll}9 & 27 \\ 1 & 10\end{array}$

Fig. 4 Frequencies of the different pachytene configurations displayed by the two iso- $\mathrm{B}^{\mathrm{L}}$ combination.

\section{Small $B$ isochromosome plus standard $B\left(1\right.$ iso- $B^{S}+$} $1 \mathrm{stB})$

The frequencies of the different pachytene configurations displayed by this combination are given in Fig. 6 . The iso- $\mathrm{B}^{\mathrm{S}}$ displays the expected symmetrical hairpin loop and the stB shows extensive self-synapsed regions in 63 per cent of the nuclei (17/27) (configuration $A$, Fig. $5 \mathrm{c})$. In the remaining nuclei ( 37 per cent) an iso$\mathrm{B}^{\mathrm{S}} \mathrm{stB}$ bivalent was observed $(\mathrm{B}, \mathrm{C}, \mathrm{D}, \mathrm{E})$. In configurations $\mathrm{B}$ and $\mathrm{E}$ there is not only homologous synapsis between the short arms of both chromosomes, but also self-synapsed regions in the stB axis and interchromosomal nonhomologous synapsis. On the other hand, bivalents corresponding to configurations $\mathrm{C}$ and $\mathrm{D}$ are probably a consequence of nonhomologous synapsis.

In this B combination there is a competitive pairing situation among the three short arms, one from the stB and the other two from the iso- $\mathrm{B}^{\mathrm{S}}$. Even if all bivalents were considered as products of homologous synapsis, their frequency is significantly lower than that expected assuming random pairing and a single distal pairing initiation site per arm (66 per cent), $\left(\chi_{1}^{2}=10.66\right.$, $P<0.01$ ).

The frequencies of one bivalent and two univalents at metaphase I were 3 per cent and 97 per cent respectively.

Small $B$ isochromosome plus two standard Bs 11 iso$\left.B^{S}+2 s t B\right)$

The frequencies of the different pachytene configurations are given in Fig. 7. Here, there is a pairing competition situation among the four short arms, two from the $s t B s$ and the other two from the iso- $\mathrm{B}^{\mathrm{S}}$. Assuming random pairing, and a single initiation site 


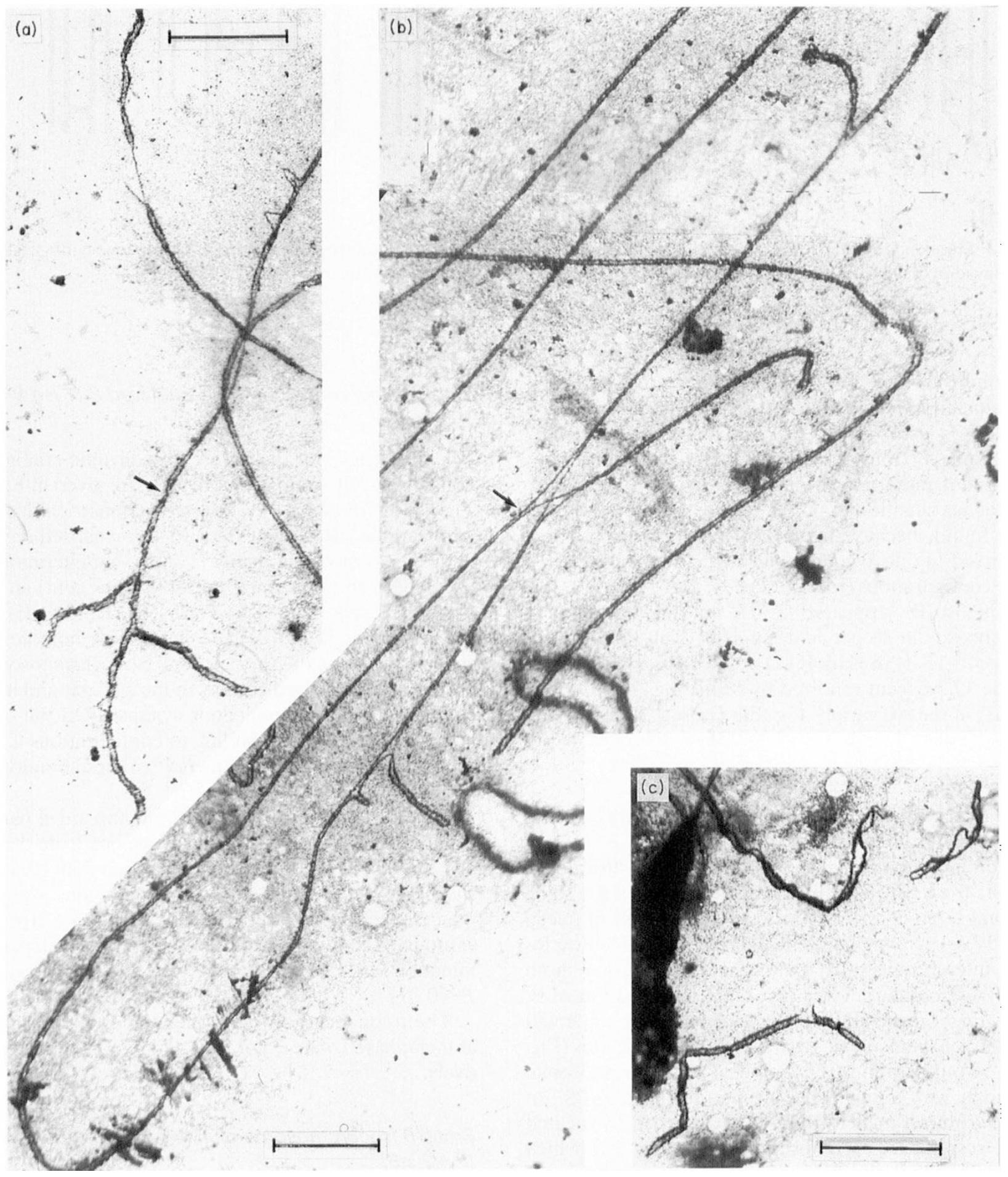

Fig. 5(a) Two iso-B ${ }^{\mathrm{L}}$; pachytene straight bivalent (arrowed) showing two regions with intrachromosomal synapsis. (b) Two iso$\mathrm{B}^{\mathrm{L}}$; pachytene bivalent (arrowed) showing interchromosomal synapsis with a single pairing partner switch asymmetrically located with respect to chromosome ends; three fold-back paired regions are also present. (c) Iso- $\mathrm{B}^{\mathrm{S}}+\mathrm{stB}$; section of a pachytene nucleus showing two $B$ univalents; iso- $B^{S}$ is almost fully paired whereas stB shows extensive self-synapsis. Each scale bar represents $5 \mu \mathrm{m}$. 
per chromosome arm, we would expect at pachytene 66.6 per cent trivalents and 33.3 per cent bivalents. However, B trivalents were only observed in two out of 10 nuclei analysed ( 20 per cent), and homomorphic bivalents appeared in the remaining eight nuclei.

The frequencies of $\mathrm{B}$ trivalents, stB bivalents and three univalents at metaphase I were 7 per cent, 62 per cent and 31 per cent, respectively.

No. nuclei

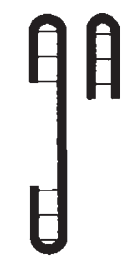

A B C

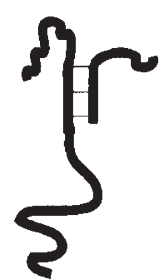$$
\text { D }
$$

1

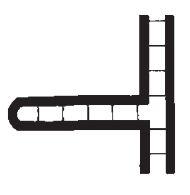

E 27

Fig. 6 Frequencies of the different pachytene configurations displayed by the iso- $\mathrm{B}^{\mathrm{s}}+\mathrm{st} \mathrm{B}$ combination.

Plant Configuration

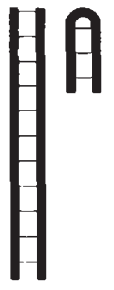

A

8

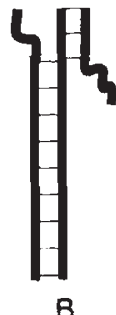

2

No. nuclei

R42

Fig. 7 Frequencies of the different pachytene configurations displayed by the iso- $B^{S}+2$ stB combination.

Plant

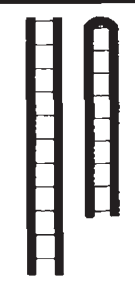

A
Configuration

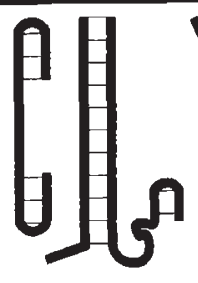

B

2.125 No. nuclei

R51 Exp. 6.934

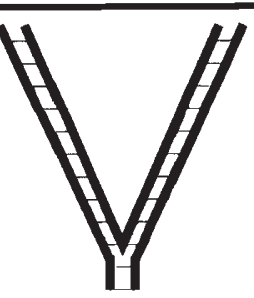

1 14.505
Large B isochromosome plus two standard Bs 11 iso$\left.B^{L}+2 s t B\right)$

The frequencies of the different pachytene configurations are given in Fig. 8. Configuration $\mathrm{A}$, that corresponds to a stB bivalent plus a fully synapsed iso- $\mathrm{B}^{\mathrm{L}}$, was observed in 41 per cent of the nuclei $(9 / 22)$. Different types of trivalents are grouped in configuration $C$ that appeared in 50 per cent of the nuclei (11) 22 ). Furthermore, an iso- $\mathrm{B}^{\mathrm{L}} \mathrm{stB}$ bivalent plus a stB univalent partially self-synapsed was observed in the remaining 9 per cent of the nuclei (B).

Pairing competition in this combination is among the four long arms, two of the stBs and the other two of the iso- $\mathrm{B}^{\mathrm{L}}$. The random-end-pairing model is usually applied to study this type of situation, however configuration $\mathrm{B}$ invalidates one of the model premises based on the effectiveness of distal initiation sites in pairing achievement.

Therefore, we have slightly modified the model. Let us denote the probabilities of the short and the long arms being paired or unpaired as $p, q, 1-p$ and 1-q, respectively. Then, the probabilities of all four long arms being paired in a pairwise fashion, only two arms being paired and all arms being unpaired will be $q^{2}$, $2 q(1-q)$ and $(1-q)^{2}$ respectively. Under these premises, the theoretical probabilities of the different chromosome configurations will be as follows.

Three univalents: $(1-p)(1-q)^{2}$.

StB bivalent plus unpaired iso- $\mathrm{B}^{\mathrm{L}}: p(1-q)^{2}$ $+(1 / 6) 2 q(1-q)(1-p)+(1 / 6) 2 p q(1-q)$.

$\mathrm{StB}$ univalents plus fully paired iso- $\mathrm{B}^{\mathrm{L}}$ :

$(1 / 6) 2 q(1-q)(1-p)$.

Iso-B ${ }^{L}$ stB bivalent: $(4 / 6) 2 q(1-q)(1-p)$.

$\mathrm{StB}$ bivalent plus fully paired iso- $\mathrm{B}^{\mathrm{L}}$ :

$(1 / 6) 2 p q(1-q)+(1 / 3)(1-p) q^{2}+(1 / 3) p q^{2}$

Trivalent: $(4 / 6) 2 p q(1-q)+(2 / 3)(1-p) q^{2}+(2 / 3) p q^{2}$.

The observed and expected frequencies of the different configurations are given in Fig. 8. Although the low number of nuclei analysed prevents a $\chi^{2}$ test, the existence of pairing preferences among the four B long arms is not evident.

The frequencies of $B$ trivalents, stB bivalents, iso$\mathrm{B}^{\mathrm{L}} \mathrm{stB}$ bivalents and three univalents at metaphase $\mathrm{I}$ were 22 per cent, 43 per cent, 21 per cent and 14 per cent, respectively.

\section{Discussion}

Most direct early prophase I observations on the behaviour of different metacentric B systems only refer to the existence of fold-back pairing to give symmetrical hairpin loops as proof of the isochromosome nature of such Bs (Müntzing \& Lima de Faria, 1949, 1953;
Fig. 8 Frequencies of the different pachytene configurations displayed by the iso- $\mathrm{B}^{\mathrm{L}}+2 \mathrm{stB}$ combination and those expected under the random-end-pairing model $(P=0.91$, $q=0.95 ;$ see text for explanation). 
Dowrick, 1952; Bosemark, 1957; Wu, 1978; Santos et al., 1993b). However, in rye plants with two large isoBs or two small iso-Bs, Müntzing \& Lima de Faria (1949, 1953) also described more complicated synaptic patterns involving not only interarm and interchromosomal pairing between the four B chromosome arms but cases of partly nonhomologous pairing too.

The results presented here confirm previous observations by Müntzing \& Lima de Faria $(1949,1953)$ and extend them because new pachytene configurations are described and their frequencies quantified. Furthermore, iso-Bs also show a peripheral location in the surface-spread nuclei and a period of pairing delayed relative to the A-set. These meiotic features are also shown by other types of rye Bs, the standard B and the deficient B (Santos et al., 1993a; Jiménez et al., 1994), and by the iso-Bs of Crepis capillaris (Jones et al., 1989). However, Fletcher \& Hewitt (1988) reported that iso-Bs of two grasshopper species pair earlier in zygotene than do the A-set.

Other than in this paper, the only iso-Bs of plants studied by surface spreading are those of $C$. capillaris (Jones et al., 1989, 1991), so comparisons between the iso-Bs from these two species can be made.

(1) Iso-Bs of rye and Crepis show anomalous pairing configurations which may reflect either the existence of reverse repeat sequences or, more likely, nonhomologous pairing. On the other hand, the meaning of the irregularities between axes (see Figs 1 and 3) remains obscure because it is difficult to ascertain whether they represent final or transient arrangements.

(2) Whereas pairing is delayed around the centromere in the iso-Bs of Crepis, no consistent pattern of synapsis initiation was observed in the iso-Bs of rye.

(3) Two B isochromosomes will give 66.6 per cent bivalents and 33.3 per cent univalents with random pairing of arms, assuming a single distal pairing initiation site per arm. The B bivalent frequencies in $C$. capillaris and rye plants were 86 per cent and 46 per cent, respectively. In the former species, it was proposed that the high frequency of bivalents was due to the occurrence of multiple pairing initiations along the isochromosome arm resulting in relatively high frequencies of PS (Jones et al., 1989, 1991). The lower than expected frequency of B bivalents in rye is quite surprising because of the greater length of these Bs compared with the Bs of $C$. capillaris $(69.6 \mu \mathrm{m}$ vs. $19.34 \mu \mathrm{m}$ ). It appears that rye iso-Bs, like stBs (Santos et al., 1993a), have no propensity for PSs, indeed, one is the maximum number of PS per 2B set (see Fig. 4). Different, although not necessarily excluding, factors such as the spatial disposition of the B isochromosomes during early prophase $\mathrm{I}$, the inactivation or nonindependence of some pairing initiation points and the nonrandom pairing of the four chromosome arms could explain this unexpected result.

(4) Iso-B bivalents, with PSs, display different synaptic configurations in these two species. In $C$. capillaris bivalents, PSs are symmetrically located with respect to chromosome ends, and interarm pairing preferentially occurs near the centromeres whereas chromosome ends show random association, that is, the ratio between interarm pairing and interchromosomal pairing is $1: 2$. In rye iso-B bivalents the only PS is sometimes $(3 / 10)$ asymmetrically located and interchromosomal pairing occurs at both putative pericentromeric and distal regions (see configuration $\mathrm{D}$, Figs 4 and $5 \mathrm{~b}$ of this work and Figs $10-12$ and 16 in Müntzing \& Lima de Faria, 1953). The underlying reasons for these specific kinds of behaviour could include differences in the number and/or location of pairing initiation sites and in the synapsis progression rates.

(5) In C. capillaris, there is a good agreement between prophase I pairing and metaphase I B associations, suggesting that a high chiasma frequency maintains pachytene configurations until metaphase $I$ as it also appears to do in Tradescantia ohiensis (Hauber, 1987). In rye, there is a reduction in the number of iso-B bivalents from pachytene to metaphase I probably due to a low chiasma frequency between $\mathrm{B}$ chromosomes.

In the plants carrying one iso- $\mathrm{B}^{\mathrm{S}}$ plus one $\mathrm{stB}$ and one iso- $\mathrm{B}^{\mathrm{S}}$ plus two $\mathrm{st} \mathrm{B}$ there is competition for pairing among the three or four short arms of the standard B. In both cases, a large predominance of selfsynapsed iso- $\mathrm{B}^{S}$ univalents was found (63 per cent and 80 per cent, respectively, Figs 6 and 7). Factors such as the bouquet disposition of the chromosomes at early prophase I, the small size of the isochromosome and the identical origin of its arms can favour interarm pairing. The increase of iso- $\mathrm{B}^{\mathrm{S}}$ univalents from pachytene to metaphase $I$ in both plants is most likely produced by a low frequency of chiasma formation in B pachytene configurations.

On the other hand, in the plant carrying one iso- $\mathrm{B}^{\mathrm{L}}$ plus two stBs no apparent pairing preferences among the four long arms were found (Fig. 8). The large size of the iso- $\mathrm{B}^{\mathrm{L}}$ and a high pairing activity of the long arm compared with that of the short arm could explain the differences between this situation and that mentioned above. In a plant of the rye variety 'Östgöta Gråråg' with this same chromosome constitution (Müntzing, 1949) the frequencies of $B$ trivalents, stB bivalents, iso$B^{L}$ stB bivalents and three $B$ univalents at metaphase I were 1 per cent, 7.8 per cent, 13.6 per cent and 70.9 per cent respectively. In 6.8 per cent of the cells the type of bivalent could not be distinguished. This author 
concluded that there was random pairing among the three chromosomes involved since homomorphic and heteromorphic bivalents fit a 1:2 ratio. In the plant analysed in this paper metaphase I frequencies of stB bivalents and iso- $\mathrm{B}^{\mathrm{L}} \mathrm{stB}$ bivalents were 43 per cent and 21 per cent, respectively, just the contrary of what Müntzing found, but no evident pairing preferences were detected at pachytene. This points to the desirability of making direct zygotene and pachytene observations of pairing because in many cases the extrapolation from metaphase I data to pachytene configurations is unfounded.

\section{Acknowledgements}

This paper was supported by a grant PB 92-0182 awarded by the Comisión Interministerial de Ciencia y Tecnología (Spain). We thank Dr R. N. Jones for reviewing this paper and for his useful comments. Agustín Fernández and the staff of the Complutense University Electron Microscope Unit, especially Dr Carlos Barba, provided valuable assistance.

\section{References}

BOSEMARK, N. o. 1957. Further studies on accessory chromosomes in grasses. Hereditas, 43, 236-297.

DIEZ, M., JIMÉNEZ, M. M. AND SANTOS, J. L. 1993. Synaptic patterns of rye B chromosomes. II. The effect of standard Bs on the pairing of the A-set. Theor. Appl. Genet., 87, 17-21.

DowRICK, G. J. 1952. The chromosomes of Chrysanthemum. I. The species. Heredity, 6, 365-375.

FLETCHER, H. L. AND HEWITT, G. M. 1988. Synaptonemal complex of univalent B chromosomes in the grasshoppers Euthrystira brachyptera and Myrmeleotettix maculatus. Heredity, 60, 383-386.

GIRALDEZ, R., CERMEÑo, M. C. AND ORELLANA, J. 1979. Comparison of $\mathrm{C}$-banding pattern in the chromosomes of inbred lines and open pollinated varieties of rye. $Z$. PflZ̈̈cht. 83, 40-48.

HAUBER, D. P. 1987. Supernumerary chromosomes in
Tradescantia I. Meiotic behaviour of three homologous isochromosomes. Genetica, 75, 117-121.

HoLM, P. в. 1986. Chromosome pairing and chiasma formation in allohexaploid wheat, Triticum aestivum, analyzed by spreading of meiotic nuclei. Carlsberg Res. Comm., $\mathbf{5 1}$, 239-294.

JIMÉNEZ, M. M., DIEZ, M. AND SANTOS, J. L. 1994. Synaptic patterns of rye B-chromosomes. III. The deficient B. Chromosome Res., 2, 93-98.

JONES, G. H., ALBINI, S. M. AND WHITEHORN, J. A. F. 1991. Ultrastructure of meiotic pairing in B chromosomes of Crepis capillaris. II. 4B pollen mother cells. Chromosoma, 100, 193-202.

JONES, G. H., WHITEHORN, J. A. F. AND ALBINI, S. M. 1989. Ultrastructure of meiotic pairing in B chromosomes of Crepis capillaris. I. One-B and two-B pollen mother cells. Genome, 32, 611-621.

JONES, R. N. AND REES, H. 1982. B Chromosomes. Academic Press, London.

LoIDL, J. 1984. Light microscopical observations on surface spread synaptonemal complexes of Allium ursinum. Caryologia, 37, 415-421.

MÜNTZING, A. 1944. Cytological studies of extra fragment chromosomes. I. Iso-fragments produced by misdivision. Hereditas, 32, 97-119.

MÜNTZING, A. 1949. The meiotic pairing of iso-chromosomes in rye. Port. Acta Biol. Ser. A . Goldschmidt vol., 831-860.

MÜNTZING, A. AND LIMA DE FARIA, A. 1949. Pachytene analysis of standard fragments and large iso-fragments in rye. Hereditas, 35, 253-268.

MÜNTZING, A. AND Lima DE FARIA, A. 1953. Pairing and transmission of a small accessory iso-chromosome in rye. Chromosoma, 6, 142-148.

SANTOS, J. L., JIMÉNEZ, M. M. AND DIEZ, M. 1993a. Synaptic patterns of rye B chromosomes. I. The standard type. Chromosome Res., 1, 145-152.

SANTOS, J. L., DEL CERRO, A., FERNANDEZ, A. AND DÍEZ, M. 1993b. Meiotic behaviour of B-chromosomes in the grasshopper Omocestus burri: A case of drive in females. Hereditas, 118, 139-143.

SANTOS, J. L., JIMÉNEZ, M. M. AND DÍEZ, M. 1994. Meiosis in haploid rye: extensive synapsis and low chiasma frequency. Heredity, 73, 580-588.

wU, T. P. 1978. Pachytene morphology of Sorghum nitidum chromosome complement. Cytologia, 43, 433-440. 